\title{
The Characteristics of Immunoglobulin and Complement by Aerobic Exercise and Stop Training
}

\author{
Qiaozhen Yan,Liping Dong,Ke Pan \\ School of Physical Education, Wenshan University, Wenshan Yunnan 663099, China
}

Key words: Immunoglobulin;Complement ; Aerobic exercise; Stop Training;Adapt

\begin{abstract}
To understand the characteristics of immunoglobulin and complement by aerobic exercise and stop training.Mthod Random selection 14 freshmen,4 weeks to aerobic exercise by treadmill $\left(9 \mathrm{Km} \cdot \mathrm{h}^{-1}\right.$,gradient is zero, $\left.15 \mathrm{~min}\right)$ for $45 \mathrm{~min}$,and aerobics every other day; Then stop training 4 weeks,phlebotomize at the weekend in 0,4,6,8, centrifugal, take supernatant,measure IgA,IgG,IgM and C3,C4's content by single radial immunodiffusion.Rsult The content of IgA,IgG,IgM and C3 decend beginning,then ascend.Conclusion Immunoglobulin and complement changed adaptively,help to improve the body's immune stystem.
\end{abstract}

\section{Introduction}

Aerobic exercise refers to exercise the human body in sufficient oxygen supply situation, including some low-intensity, prolonged exercise, such as: aerobics, square dancing, jogging, etc. ${ }^{[1]}$.Adhere to long-term aerobic exercise can improve cardiovascular and lung function, improve immune system function, enhance resistance. Immunoglobulins can neutralize bacterial toxins, blocking pathogen invasion response play a very important role in the face of specific pathogenic microorganisms ${ }^{[2]}$. Complement is also widely Caiyu microbial organism defense response and immune regulation, immune response to injury also may be connected to pathology. Current research on the immune system of aerobic exercise more, and stop training for the change of the few studies of the immune system. Based on the Xi'an Institute of Human Movement Science 14 freshmen aerobic endurance training, detection of aerobic exercise and body serum IgA, IgG, IgM and complement stop training of C3, C4 changes in activity, further provided with aerobic exercise to enhance physical fitness, improve immunity provides a theoretical basis, the theoretical basis for the further understanding of the impact of aerobic exercise on the immune system.

\section{Subjects and Methods}

\subsection{The study}

Human subjects were Xi'an Institute of Physical Science Grade 14 boys, mean age $19.5 \pm 0.7$ years, mean height $1.75 \pm 08 \mathrm{~m}$, the average weight of $64.40 \pm 3.35 \mathrm{~kg}$, health, sports, without any professional training.

2.2 Research Methods

\subsection{1 sports mode}

Subjects in the active treadmill ( $9 \mathrm{Km} \cdot \mathrm{h}-1$, the slope is 0 ) on the total time of 45 minutes of aerobic exercise, a total of eight weeks of aerobic exercise every other day.

2.2.2 Test schedule

0,4,6,8 weeks after the end of one hour of aerobic exercise on weekends blood, every male venous 
blood $4 \mathrm{ml}$, separation of serum, the standby detection of immunoglobulin and complement relevant indicators.

2.2.3 Main indicators and methods

(1)Determination of IgA, IgG, IgM: the use of serum IgA, IgG, IgM content, single expanded edition of the Shanghai Cooperation Jie door biotechnology products, measurement step unidirectional radial immunodiffusion according to detailed instructions.

(2) C3 and C4 are measured: C3 and C4 for an amount of serum immunoglobulins and different, the other steps with immunoglobulins.

2.2.4 Test apparatus and materials

(1)Quinton 5500 treadmill-type activities (United States)

(2)CD 1600 automatic hematology analyzer (Japan)

(3) Polar 3000 (Finland)

(4)XF IItype centrifugal sedimentation machine (Shanghai M edical Instrument Factory)

(5)IgA, IgG, IgM detection reagent single radial immunodiffusion plate (Shanghai Je door Biotechnology Cooperation)

6) C3, C4 single radial immunodiffusion plate detection reagents (Shanghai Je door Biotechnology Cooperation)

\section{Data Processing}

Using Execl package for statistical, each measurement result $\overline{\mathrm{X}} \pm \mathrm{s}$, before and after the group using $\mathrm{t}$ test, comparison between groups using analysis of variance, with $\mathrm{p}<0.05$ for the difference was statistically significant level, $\mathrm{p}<0.01$ for the difference was statistically significant level.

\section{Results}

3.1 Adaptations of aerobic exercise plus immunoglobulin stop training of

From Table 1, after performing aerobic exercise four weeks, IgA, IgG, IgM have varying degrees of decline, in which IgG, IgM decline more apparent than in the previous exercise, there was a significant difference $(\mathrm{P}<0.01)$, IgA, there are significant differences $(\mathrm{P}<0.05)$ than in the previous campaign. 4 weeks after the end of aerobic exercise, stop training two weeks, IgA, IgG, IgM content have varying degrees of increase, slightly lower than the content before exercise. Stop training for 4 weeks, the content of IgA, IgG, IgM continues to increase, which has more than IgG content before exercise.

Table 1: Effect of aerobic exercise + stop training immunoglobulin activity (unit:g/L)

\begin{tabular}{ccccc}
\hline & WO & W4 & W6 & W8 \\
\hline IgA & $2.42 \pm 0.31$ & $2.19 \pm 0.46^{*}$ & $2.39 \pm 0.26 \#$ & $2.41 \pm 0.27 \#$ \\
IgG & $9.47 \pm 0.93$ & $8.32 \pm 1.09 * *$ & $9.34 \pm 1.38 \#$ & $9.54 \pm 1.47 \# \#$ \\
IgM & $1.24 \pm 0.13$ & $1.07 \pm 0.28 * *$ & $1.17 \pm 0.25 * \#$ & $1.19 \pm 0.24 \#$
\end{tabular}

Note: Compared to Week 0, * represents $\mathrm{P}<0.05$, ** indicates $\mathrm{P}<0.01$; compared with the first four weeks, \# indicates $\mathrm{P}<0.05$, \#\# indicates $\mathrm{P}<0.01$

3.2 adaptations complement system to stop training of aerobic exercise plus

Table 2 shows that, after four weeks of aerobic exercise, C3 content lower than the previous motion, and there is a significant difference $(\mathrm{P}<0.05)$ compared to the previous exercise, $\mathrm{C} 4$ levels than before exercise increased. Stop training after 2 weeks, an increase in the content of C3, and C4 
levels decrease, stop training after 4 weeks C3 and C4 content and stop training two weeks are substantially equal.

Table 2: Effect of aerobic exercise + stop training system complement activity (unit: g / L)

\begin{tabular}{ccccc} 
& WO & W4 & W6 & W8 \\
\hline C3 & $1.25 \pm 0.27$ & $0.82 \pm 0.21^{*}$ & $1.22 \pm 0.25 \#$ & $1.22 \pm 0.13 \#$ \\
4 & $0.46 \pm 0.03$ & $0.47 \pm 0.07$ & $0.46 \pm 0.09$ & $0.46 \pm 0.06$
\end{tabular}

Note: Compared to Week 0, * represents $\mathrm{P}<0.05$, ** indicates $\mathrm{P}<0.01$; compared with the first four weeks, \# indicates $\mathrm{P}<0.05$, \#\# indicates $\mathrm{P}<0.01$

\section{Analysis and discussion}

5.1 Adaptations of aerobic exercise plus immunoglobulin stop training of

Antibody is antigenic material after stimulation of the formation of the body, having a function of binding reaction with the antigen-specific immunoglobulin, an antibody having the activity or the chemical structure of an antibody immunoglobulin becomes similar globulin (immunoglobulin, Ig) . Immunoglobulin ubiquitous in mammals and human blood, tissue fluid and the outer fluid secretion, a protein of continuity, immunoglobulin structure determines its function, has to recognize a specific antigen binding, activation of complement, binding to the cell selective transmission, staphylococcal protein A binding and biological activity. It has been found in human immunoglobulin immunoglobulin G (IgG), immunoglobulin A (IgA), immunoglobulin M (IgM), etc., which have their own unique characteristics and fundamental role.

(1) IgG

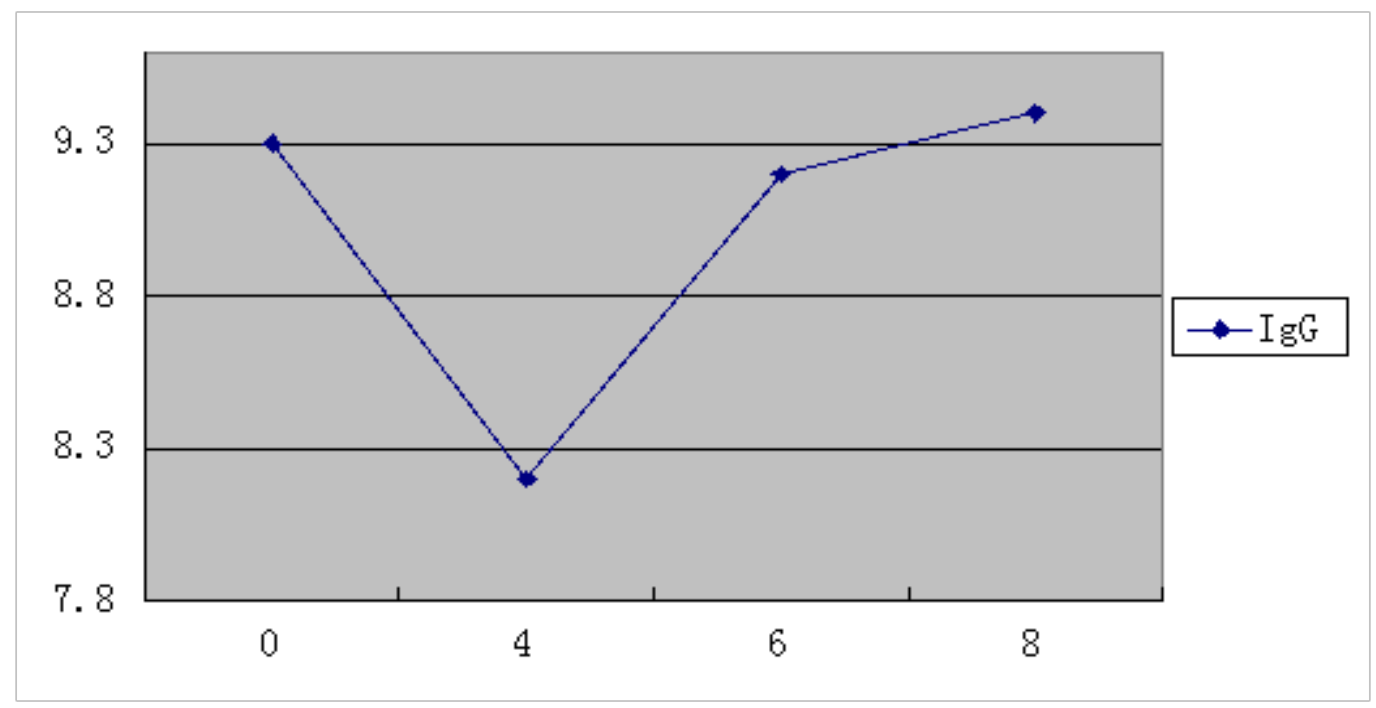

Figure 1: Adaptive Changes of IgG to stop training plus aerobic exercise

IgG is the major component in human serum Ig, the content is high, wide distribution, mostly monomeric protein, the primary immune response is the most durable and most important antibody. It is easier than other Ig through the capillary walls, diffuse into the tissue space, play a dominant role in the anti-infection. Most anti-bacterial, anti-toxic and anti-viral antibodies belonging to IgG, can promote phagocytosis monocyte-macrophage cells, and viruses and neutralize toxins. In this study, by four weeks of aerobic exercise and then stop training four weeks, IgG declining trend (Figure 1) after the end of the aerobic exercise, which may be subject and never participated in physical exercise every other day for 45 minutes of aerobic load movement is relatively large subjects, making them non-specific mechanisms of functioning of the body is 
reduced, and inspection results before the data is not the same, may be associated with the test program, the differences related to movement, but prolonged or high intensitythe movement for the body's immune system but has a negative impact, it will lead to suppression of the immune response, increasing susceptibility source of acute infectious diseases, reduce the body's resistance to infection of immune function. 4 weeks after the end of the subject respiratory infections, diarrhea and other diseases, the prevalence was 10\%. Increase stop training two weeks after more significantly, stop training gradually increased after 4 weeks, two weeks before the amplitude ratio decreased after stop training, the subjects immune function gradually recovered, the proportion of infectious diseases is gradually reduced.

\section{(2) IgA}

Serum and secretory IgA is divided into two kinds, IgA than IgG synthesis rate is slightly lower, faster decomposition, short half-life, low levels in serum. Serum is mainly in monomeric form, but also dimers, primarily from mesenteric lymph tissue plasma cells. The outer body secretions mainly dimeric IgA dominant, respiratory tract, gastrointestinal tract, urogenital tract lamina propria, etc. in the plasma cells, the body's mucosal defense system is the main component. IgA has significant anti-bacterial, anti-viral and anti-toxin function, it can with tissue antigen protein or antigen binding has a special force to avoid this type of antigen into the circulation. IgA secretion out more content, coupled with its characteristic structure, not easy to be destroyed ships protease, so that it can maintain its immune activity in the local mucosal surface, anti-infective, anti-allergy an important immune barrier. The experiments to study adaptive changes of IgA in this process (Fig.2) by four weeks and four weeks of aerobic exercise training stopped after four weeks of aerobic exercise ended, IgA a downward trend, after they stop training IgA contentgradually increased. This might exercise in subjects with a great relationship, studies have shown, can instantly reduce the level of saliva SigA after exercise, and strength training lead to chronic high SigA sustainable period of time, this experiment could be somewhat larger exercise intensity is suppressed and the subject of the body's mucosal immune function.

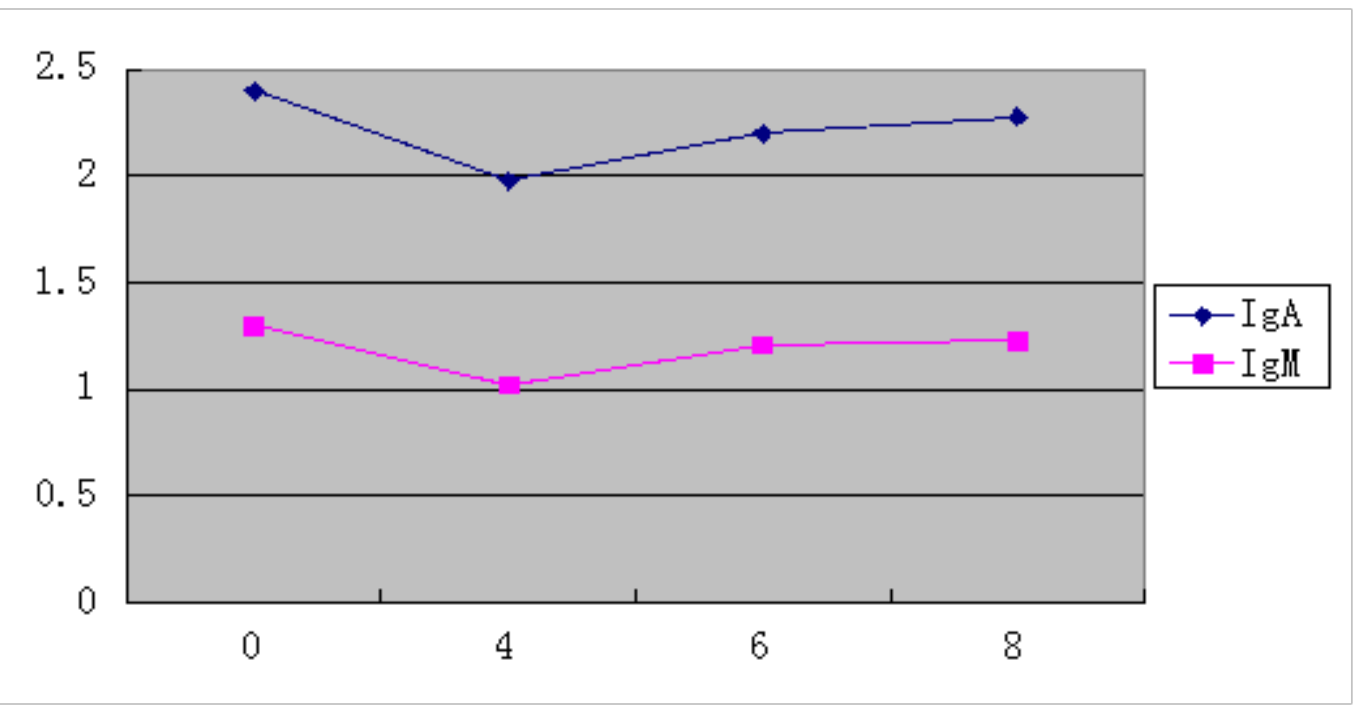

\section{(3) $\operatorname{Ig} M$}

Figure 2:Adaptations IgA, IgM to stop training plus aerobic exercise

During the early ontogeny of IgM antibody synthesis, synthesis of the main venues are the spleen, due to high molecular weight can not through the vessel wall, so almost all of IgM synthesis in the blood. IgM antibody belongs to a virus, there is the participation of the complement system, can destroy tumor cells, it is a high-performance bio-antibody. Changes in this experiment IgM and IgA were similar (Fig.2), declined at the end of aerobic exercise, gradually increase the amount after the 
stop training, this exercise intensity and exercise frequency and have a great relationship.

In this experiment IgA, IgM, IgG in the fourth week have both been dropped, then gradually picked up as an exercise load stress, the body gradually adapt to the transition from not suited to this experiment Ig activity, there have been similar changes process.Liu Ruijiang ${ }^{[3]}$ through the 12 weeks of Taijiquan found that the content of IgA did not significantly change the content of IgG and IgM have increased significantly. Wang et al ${ }^{[4]}$ by Taiji training, the content of serum IgG female college students than the control group, and IgM, IgA was no significant difference. Liu Yan ring ${ }^{\text {[5] }}$ found that tai chi exercise can improve female students IgA and IgG levels. Nieman et al ${ }^{[6]}$ found that after prolonged exercise serum Ig activity were significantly increased. Through 12 weeks after exercise of moderate intensity Ig activity are significant changes in Mitchell et al ${ }^{[7]}$.

5.2 adaptations complement system to stop training of aerobic exercise plus

Complement is not a single molecule, is a group of proteins with enzymatic activity present in the serum and tissue fluids of a heat-activated later. Under normal circumstances, the complement in body fluids was inactive state, when subjected to some kind of "activator", the ingredients are sequentially activated, the activation of complement components or cleavage fragment having enzyme activity. Complement in the body involved in specific and non-specific immune response, sterilization, cytolytic and fire-viral effects. Regulations can also complement the role of non-specifically promote phagocytic effect, the body is an important aspect of anti-infection immunity. The core component of the complement system is C3 protein, is a key component of the system exert effector functions, C3 is the biologically active form of the protease cleavage product. Classical and alternative pathways activated in different ways including different protein components and, thus, became the C3 convertase enzymes. C4 is the classical pathway can be activated a second special soluble serum proteins.

The present study examined the highest concentrations in the serum of the most important complement component $\mathrm{C} 3$ and $\mathrm{C} 4, \mathrm{C} 3$ found in the stress response generated by the game has also undergone a change. C3 as the exercises will be a gradual upward trend (Figure 3), which may be due to an immunoglobulin to kill pathogens, must first and Complement C3 binding to play a role, therefore, both in vivo trends should basically the same. In addition, most of the immunoglobulin is a cytokine produced by the body after receiving specific antigen stimulation by the secretion activated B cells, and therefore stress cytokine production because the body is affected, so that the activated B cells are affected; affected B-cell activation, such thatpROLIFERATION affected; proliferation itself is affected, making immunoglobulin secretion affected; immunoglobulin secretion affected, so that complement and generate activity affected. This is an immunoglobulin and complement symbiotic relationship of the decision. Jiang Guifeng ${ }^{[8]}$ and other observed changes in the body's complement of female students by Aerobics, studies show exercise three times in a week, from 8 weeks can improve the body's complement activity. Feng Yi Chong ${ }^{[9]}$ and other studies conducted by the Health Qigong Yi Jin Jing immune function in the elderly, found that by Yi Jin Jing exercises, CD4+,CD8+,CD4+/CD8+ ratio were significantly increased. Wang Mei ${ }^{\text {[10] }}$ through aerobic exercise and Qigong research on immune function in older women found that each group CD8 after high-intensity exercise immediately rise. Wan Wenjun ${ }^{[11]}$ and so on through the study found that adaptive changes after Intensive winter training C3 and C4 produced mixed, C3 decreased, and C4 levels were increased.

\section{Conclusions}

The study found that by four weeks of aerobic exercise +4 weeks stop training, Ig content in serum decreased after aerobic exercise ended, after rising gradually stop training for the activity of the 
complement system also have some impact.

\section{References}

[1] Qiping Huang,Guifeng Jiang et. Effect of Aerobics Exercise on Female College serum immunoglobulin and complement [J] Beijing Sport University .2005,28 (12): 1649-1651.

[2] EliakimA, Wolach B, Kodesh E, et al.Cellular and humoral immune re-sponse to exercise among gymnasts and untrained girls[J]. Int J Sports Med. 1997, Apr;18(3):8-12.

[3] Ruijiang Liu. Tai Chi exercise on immune balance of non-sports Major Female University Students [J]. SPORTS SCIENCE .2013,35 (5): 87-89.

[4] Wang Y T,Taylor L,Pearl M,et al. Effects of Tai Chi exercise on physical and mental health of college students[J]. Am J Chin Med,2004,32(3): 453-459.

[5] Liu Yan ring, Nie Hua, Ma Guodong et. Effects of Taijiquan and upper respiratory tract infection and immune globulin Female College Students relations [J]. Beijing Sports University, 2005,25 (8): 1089-1090.

[6] Nieman DC,Pedersen BK.Exercise and immune function:Recent developments[J].Int J Sports Med,1999,27(2):73-80.

[7] Mitchell JB,Paquet AJ,Pizza FX,et al. The effect of moderate aerobic training on lymphocyte proliferation[J]. IntJSports Med,1996,17(5): 384-389.

[8] Guifeng Jiang, Huang-Qiping et. Aerobics on Body complement activity [J]. Tianjin Institute of Physical Education, 2005,20(5):76-78.

[9] Yichong Feng,GuoLin Zhong and et. Health Qigong Yi Jin Jing on immune function in the elderly [J]. Jilin Institute of Physical Education, 2013,29 (5): 68-70 .

[10]Wang-fengmei, Yang Yi. Effects of Aerobic Exercise Qigong and immune function in elderly women [J]. Wuhan Institute of Physical Education, 2006,40(7):47-50.

[11]Wenjun Wan,Xuanmin Hao.Exercise training on immune function and immune effects[J].JOURNAL sports,2012,19(4):140-144. 